\title{
Overview of ASDEX Upgrade Results
}

A. Kallenbach, J. Adamek ${ }^{1}$, L. Aho-Mantila ${ }^{2}$, S. Äkäslompolo ${ }^{2}$, C. Angioni, C.V. Atanasiu ${ }^{3}$, M. Balden, K. Behler, E. Belonohy, A.

Bergmann, M. Bernert, R. Bilato, V. Bobkov, J. Boom, A. Bottino, F.

Braun, M. Brüdgam, A. Buhler, A. Burckhart, A. Chankin, I.G.J.

Classen $^{4}$, G.D. Conway, D.P. Coster, P. de Marné, R. D'Inca, R. Drube, R.

Dux, T. Eich, N. Endstrasser, K. Engelhardt, B. Esposito ${ }^{5}$, E. Fable, H.-U.

Fahrbach, L. Fattorini ${ }^{6}$, R. Fischer, A. Flaws, H. Fünfgelder, J.C. Fuchs,

K. Gál ${ }^{7}$, M. García Muñoz, B. Geiger, M. Gemisic Adamov, L. Giannone,

C. Giroud $^{8}$, T. Görler, S. da Graca ${ }^{6}$, H. Greuner, O. Gruber, A. Gude, S.

Günter, G. Haas, A.H. Hakola ${ }^{2}$, D. Hangan, T. Happel ${ }^{9}$, T. Hauff, B.

Heinemann, A. Herrmann, N. Hicks, J. Hobirk, H. Höhnle ${ }^{10}$, M. Hölzl,

C. Hopf, L. Horton ${ }^{11}$, M. Huart, V. Igochine, C. Ionita ${ }^{12}$, A. Janzer, F.

Jenko, C.-P. Käsemann, S. Kálvin ${ }^{7}$, O. Kardaun, M. Kaufmann, A.

Kirk $^{8}$, H.-J.Klingshirn, M. Kocan, G. Kocsis ${ }^{7}$, H. Kollotzek, C. Konz, R.

Koslowski $^{13}$, K. Krieger, T. Kurki-Suonio ${ }^{2}$, B. Kurzan, K. Lackner, P.T.

Lang, P. Lauber, M. Laux, F. Leipold ${ }^{14}$, F. Leuterer, A. Lohs, N.C.

Luhmann, Jr. ${ }^{15}$, T. Lunt, A. Lyssoivan ${ }^{16}$, H. Maier, C. Maggi, K. Mank,

M.-E. Manso ${ }^{6}$, M. Maraschek, P. Martin ${ }^{17}$, M. Mayer, P.J. McCarthy ${ }^{18}$,

R. McDermott, H. Meister, L. Menchero, F. Meo ${ }^{14}$, P. Merkel, R. Merkel,

V. Mertens, F. Merz, A. Mlynek, F. Monaco, H.W. Müller, M. Münich, H.

Murmann, G. Neu, R. Neu, B. Nold ${ }^{10}$, J.-M. Noterdaeme, H.K. Park ${ }^{19}$,

G. Pautasso, G. Pereverzev, Y. Podoba, F. Pompon, E. Poli, K. Polochiy, S.

Potzel, M. Prechtl, M.J. Püschel, T. Pütterich, S.K. Rathgeber, G. Raupp,

M. Reich, B. Reiter, T. Ribeiro, R. Riedl, V. Rohde, J. Roth, M. Rott, F.

Ryter, W. Sandmann, J. Santos ${ }^{6}$, K. Sassenberg ${ }^{18}$, P. Sauter, A.

Scarabosio, G. Schall, K. Schmid, P.A. Schneider, W. Schneider, G.

Schramm, R. Schrittwieser ${ }^{12}$, J. Schweinzer, B. Scott, M. Sempf, F.

Serra $^{6}$, M. Sertoli, M. Siccinio, A. Sigalov, A. Silva ${ }^{6}$, A.C.C. Sips ${ }^{11}$, F.

Sommer, A. Stäbler, J. Stober, B. Streibl, E. Strumberger, K. Sugiyama,

W. Suttrop, T. Szepesi ${ }^{7}$, G. Tardini, C. Tichmann, D. Told, W. Treutterer, L. Urso, P. Varela ${ }^{6}$, J. Vincente ${ }^{6}$, N. Vianello ${ }^{17}$, T. Vierle, E. Viezzer, C. Vorpahl, D. Wagner, A. Weller, R. Wenninger, B. Wieland, C. Wigger, M. Willensdorfer $^{20}$, M. Wischmeier, E. Wolfrum, E. Würsching, D. Yadikin, Q. Yu, I. Zammuto, D. Zasche, T. Zehetbauer, Y. Zhang, M. Zilker, H. Zohm

Max-Planck-Institut für Plasmaphysik, EURATOM Association, Garching, Germany 
${ }^{1}$ Institute of Plasma Physics, Praha, Czech Republic

2 Asscociation EURATOM-Tekes, Helsinki, Finland

${ }^{3}$ Institute of Atomic Physics, EURATOM Association-MEdC, Romania

${ }^{4}$ FOM-Institute for Plasma Physics Rijnhuizen, EURATOM Association, TEC, Nieuwegein, The Netherlands

${ }^{5}$ C.R.E ENEA Frascati, EURATOM Association, CP 65, 00044 Frascati, (Rome), Italy

${ }^{6}$ CFN, EURATOM Association-IST Lisbon, Portugal

${ }^{7}$ KFKI, EURATOM Association-HAS, Budapest, Hungary

${ }^{8}$ EURATOM/CCFE Fusion Association, Culham Science Centre, UK

${ }^{9}$ Ciemat, Madrid, Spain

${ }^{10}$ Institut für Plasmaforschung, Universität Stuttgart, Germany

${ }^{11}$ EFDA-JET, Culham, United Kingdom

${ }^{12}$ University of Innsbruck, EURATOM Association-AW, Austria

${ }^{13}$ Forschungszentrum Jülich, Germany

${ }^{14}$ Risoe, EURATOM Association-RISOE, Roskilde, Denmark

15 University of California at Davis, Davis, CA 92186-5608, USA

${ }^{16}$ LPP-ERM/KMS, EURATOM Association-Belgian State, Brussels, Belgium

${ }^{17}$ Consorzio RFX, EURATOM Association-ENEA, Padova, Italy

${ }^{18}$ Physics Department, University College Cork, Association EURATOM-DCU, Ireland

${ }^{19}$ POSTECH, Pohang, Gyeongbuk, 790-784, Korea

${ }^{20}$ IAP, TU Wien, EURATOM Association-AW, Austria

E-mail: Arne.Kallenbach@ipp.mpg.de

\begin{abstract}
The ASDEX Upgrade programme is directed towards physics input to critical elements of the ITER design and the preparation of ITER operation, as well as addressing physics issues for a future DEMO design. After the finalization of the tungsten coating of the plasma facing components, the re-availability of all flywheel-generators allowed high-power operation with up to $20 \mathrm{MW}$ heating power at $\mathrm{I}_{p}$ up to 1.2 MA. Implementation of alternative ECRH schemes (140 GHz O2- and X3-mode) facilitated central heating above $\mathrm{n}_{e}=1.2 \cdot 10^{20}$ $\mathrm{m}^{-3}$ and low $\mathrm{q}_{95}$ operation at $\mathrm{B}_{t}=1.8 \mathrm{~T}$. Central O2-mode heating was successfully used in high P/R discharges with $20 \mathrm{MW}$ total heating power and divertor load control with nitrogen seeding. Improved energy confinement is obtained with nitrogen seeding both for type-I and type III ELMy conditions. The main contributor is increased plasma temperature, no significant changes of the density profile have been observed. This behaviour may be explained by higher pedestal temperatures caused by ion dilution in combination with a pressure limited pedestal and hollow nitrogen profiles. Core particle transport simulations with gyrokinetic calculations have been benchmarked by dedicated discharges using variations of the ECRH deposition location. The reaction of normalized electron density gradients to variations of temperature gradients and the $\mathrm{T}_{e} / \mathrm{T}_{i}$ ratio could be well reproduced. Doppler reflectometry studies at the L-H transition allowed the disentanglement of the interplay between the oscillatory geodesic acoustic modes (GAMs), turbulent fluctuations and the mean equilibrium ExB flow in the edge negative $\mathrm{E}_{r}$ well region just inside the separatrix. Improved pedestal diagnostics revealed also a refined picture of the pedestal transport in the fully developed $\mathrm{H}$-mode type-I ELM cycle. Impurity ion transport turned out to be neoclassical in between ELMs. Electron and energy transport remain anomalous, but exhibit different recovery time scales after an ELM. After recovery of the pre-ELM profiles, strong fluctuations develop in the gradients of $\mathrm{n}_{e}$ and $\mathrm{T}_{e}$. The occurrence of the next ELM cannot be explained by the local current diffusion time scale, since this turns out to be too short. Fast ion losses induced by shear Alfvén eigenmodes have been investigated by time-resolved energy and pitch angle measurements. This allowed the separation of the convective and diffusive loss mechanisms.
\end{abstract}




\section{Introduction and technical boundary conditions}

ASDEX Upgrade (AUG) is operated with full tungsten coated plasma facing components since its 2007 experimental campaign [1]. After initial campaigns demonstrated plasma operation with pure tungsten plasma facing components (PFCs) without wall conditioning, phases with boronization intervals of 4-6 weeks were run in order to reduce the intrinsic impurity content, in particular that of $\mathrm{O}$ and $\mathrm{C}$ [2]. The lower intrinsic impurity content was found to reduce tungsten sputtering rates and therefore to widen the operational range. This domain is partly restricted by the occurrence of central tungsten accumulation in combination with electron density profile peaking [3]. The coverage of tungsten by boron layers appears to be a short term effect (less than 2 experimental days) for the erosion dominated outer divertor and main chamber wall surfaces, as can be shown by comparison of tungsten fluxes and concentrations measured in boron-free and boronized discharges. After repair of the flywheel generator 4 (EZ4), which was damaged in April 2006, full power and energy supply became available again in October 2009. In fact, retaining some of the measures taken before for the better exploitation of the limited power supply, peak power and maximum energy for ASDEX Upgrade operation are increased now as compared to the values which were in place before the EZ4 damage. In particular for low ELM frequency conditions, operation with tungsten plasma facing components (PFCs) requires a few MW central heating to prevent central tungsten accumulation. The use of ICRF for this purpose was found to be limited due to strong tungsten sputtering at the antenna limiters [4]. Improved antenna designs are currently under investigation. A first design change will be tested for one antenna in autumn 2010 [5]. Central ECRH with $140 \mathrm{GHz}$ in the standard X2 scheme (second harmonic, extraordinary polarisation) at a toroidal field $\mathrm{B}_{t}=-2.5 \mathrm{~T}$ is restricted by the density cut-off, which occurs for peaked electron densities at $1 \mathrm{MA}$ discharges and even for quite flat $\mathrm{n}_{e}$ profiles at higher currents. To allow for central heating at high currents and low $\mathrm{q}_{95}$ operation at reduced toroidal field, O2-mode (second harmonic, ordinary polarisation) heating at $\mathrm{B}_{t}=-2.5 \mathrm{~T}$ and $\mathrm{X} 3$-mode heating (third harmonic) at $\mathrm{B}_{t}=-1.8 \mathrm{~T}$ have been developed [6]. Both schemes have a reduced single-pass absorption which potentially endangers in-vessel components due to ECR stray radiation. This problem has been solved by utilizing the $\mathrm{X} 2$ absorption on the high field side for the central X3 scheme. For the $\mathrm{O} 2$ scheme, a holographic reflector was installed at the inner wall which reflects the non-absorbed ECR radiation back through the plasma center and towards the robust passive stabilizing loop at the low field side. To protect the target tungsten coating from thermal overload in high power discharges, feedback-controlled nitrogen seeding has routinely been used for discharges with more than $10 \mathrm{MW}$ of heating power. This paper summarises the experimental results obtained in ASDEX Upgrade during the autumn 2008 and 2009 campaigns. 


\section{Pedestal physics}

\subsection{Inter-ELM structure of the pedestal}

The pedestal is the key area for the total stored energy as well as the impurity content of H-mode plasmas in AUG. While for the energy content temperature profile stiffness is the dominant mechanism which propagates pedestal parameters to the core plasma, the impurity content is determined by the interplay of edge impurity sources and the out-flushing of impurities from the pedestal by ELMs. Several diagnostics improvements allowed us to refine

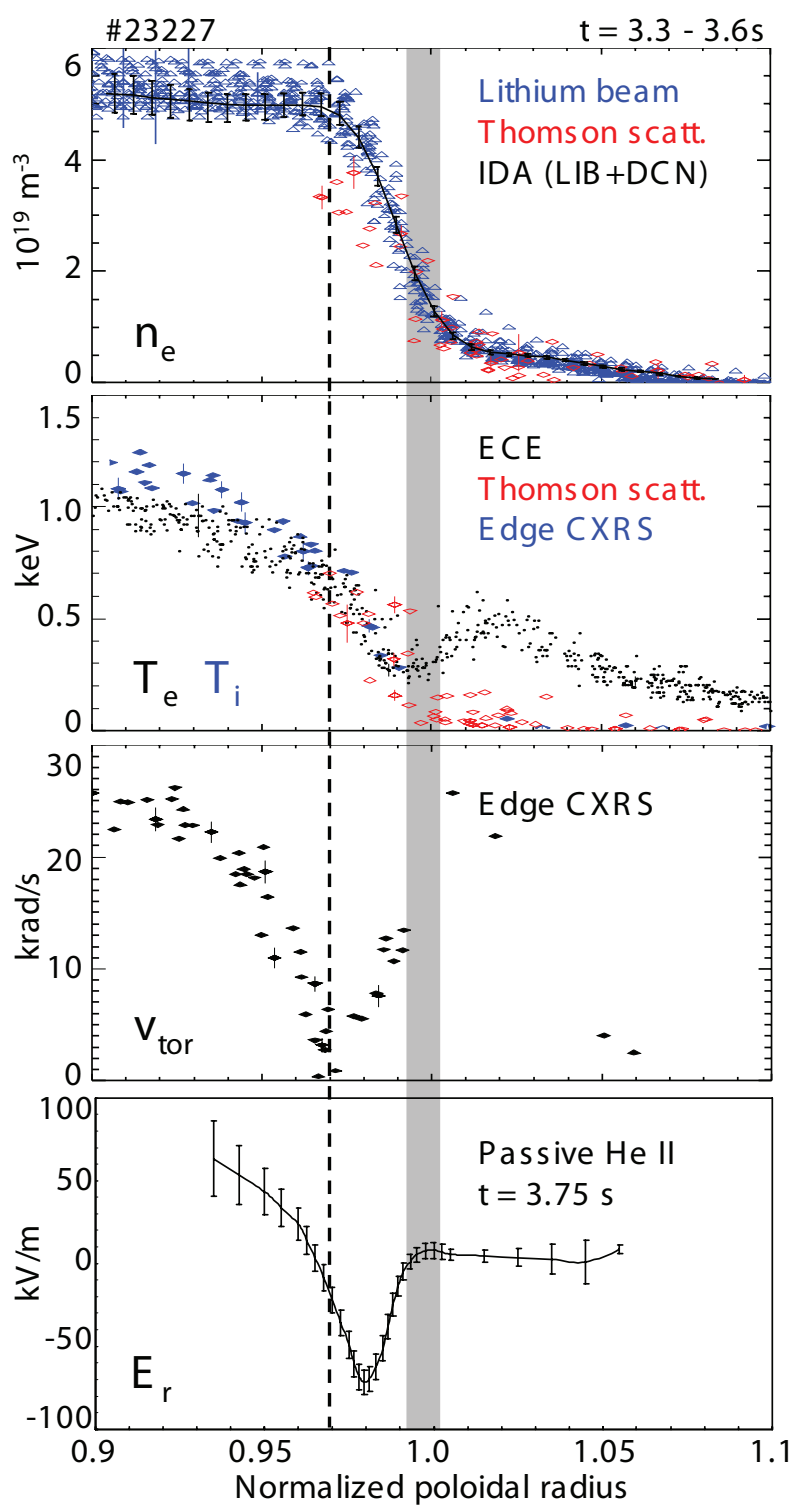

Figure 1. Pedestal profiles of the electron density (the solid line shows the result of integrated data analysis (IDA) of Lithium beam and DCN interferometer data), electron and ion temperature, toroidal angular rotation frequency and radial electric field for the inter-ELM phase of a type-I ELMy H-mode.

the picture of the spatial pedestal structure and its temporal evolution during an ELM cycle 
[7]. ELM-synchronized spatial profiles of various parameters during the inter-ELM phase are shown in figure 1 [8]. Only data which lie in the time window of -3.5 to $-0.5 \mathrm{~ms}$ before an ELM

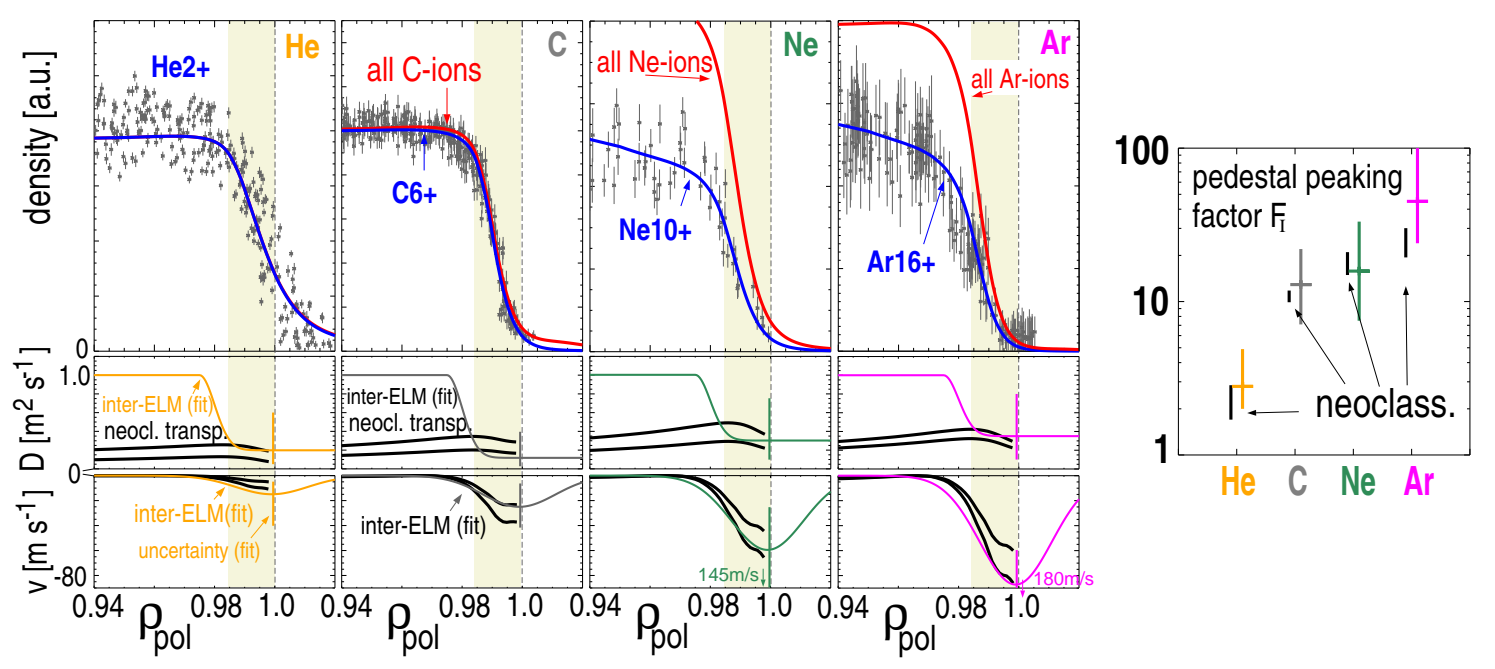

Figure 2. Pedestal profiles of impurities with different charge $Z$ measured by CXRS and STRAHL modelling of the corresponding transport coefficients D,v. Neoclassical values are shown for comparison. The right figure shows the experimental ETB impurity peaking factor between separatrix and pedestal top versus the neoclassical predictions for the different species.

are chosen, while the typical ELM period was $15 \mathrm{~ms}$. A radial shift of the plasma by $2.5 \mathrm{~cm}$ was employed to improve the spatial resolution. The most remarkable features are a negative peak of the toroidal rotation profile situated at the location of the density pedestal top as well as a pronounced negative dip of the radial electric field profile situated in the steep gradient zone. The $\mathrm{E}_{r}$ profile shown in figure 1 has been derived from the (mainly poloidal) Doppler shift of passive HeII emission from the plasma edge. Its magnitude and spatial position corresponds in good approximation to the $\nabla p / n$ term in the radial force balance of the deuterium ions [9]. It has to be noted here that the radial electric fields derived from Doppler reflectometry measurements [10] show the same spatial structure (width and position of minimum), but for certain $\mathrm{H}$-mode conditions systematically lower values of $\mathrm{E}_{r}$. A possible reason may be the fact that Doppler reflectometry needs some degree of turbulence for backscattering which might be too low in high performance $\mathrm{H}$-modes. Generally, the measured $\mathrm{E}_{r}$ corresponds to a low perpendicular velocity of the fuel ions, i.e. their ExB rotation velocity approximately cancels their diamagnetic drift velocity. This behaviour is consistent with predominantly neoclassical ion transport in the edge transport barrier (ETB) region.

\subsection{Impurity transport in the edge transport barrier region}

Further evidence of neoclassical ion transport in the ETB region is obtained from the analysis of radial profiles of different impurity species in the edge transport barrier region measured by the edge charge exchange recombination diagnostic, as shown in figure 2 [11]. Diffusion coefficient $\mathrm{D}$ and convective (inward) velocity $\mathrm{v}$ were obtained by a fit of the time dependent 


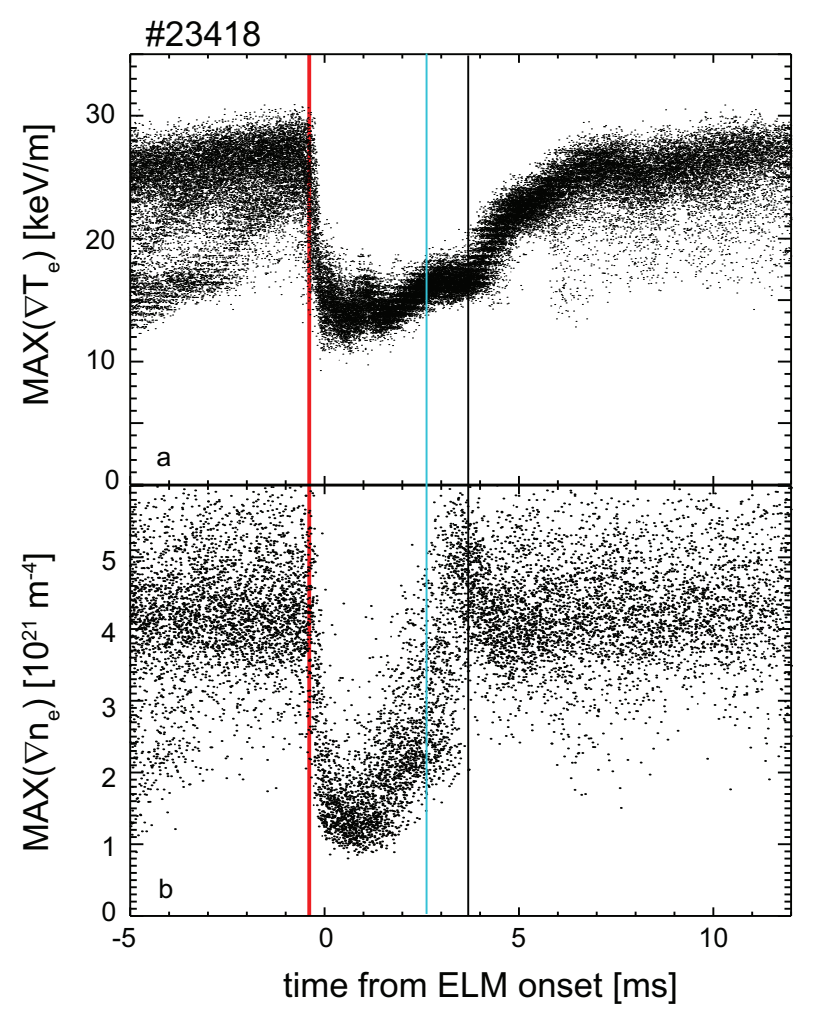

Figure 3. Maximum gradients of the electron temperature and density during an ELM cycle, measured by ECE and combined Li-beam/interferometry, respectively. Data represent the coherent overlay of 105 ELM cycles.

profile changes during an ELM cycle with the impurity transport code STRAHL [12]. Also shown are the ETB impurity peaking factors (relative rise of the impurity density from the separatrix up to the pedestal top). Quantitative agreement is found between the fitted transport coefficients and the neoclassical prediction, resulting in a pronounced impurity density rise between separatrix and pedestal top. Obviously, for high-Z impurities an additional, outward directed transport mechanism is required to avoid excessive core concentrations. ELM flushing has been identified as a corresponding mechanism in the standard H-mode [12]. For conditions of low ELM frequency or full ELM stabilization, an additional outward impurity ion transport mechanism has to be maintained to limit the pedestal high-Z impurity concentration, like, e.g., the edge harmonic oscillation (EHO) in the QH-mode [13].

\subsection{Transport variations during the ELM cycle}

The improved diagnostics also allowed the refinement of the picture of transport and stability of the pedestal region during an ELM cycle [7]. Figure 3 shows the temporal evolution of the maxima of the electron temperature and density gradients over an ELM cycle. The recovery of the gradients can be divided into different phases. After the ELM crash, first the $\mathrm{T}_{e}$ gradient recovers moderately. Then, it stays at a constant level while the density gradient rises. When the density gradient has recovered its pre-ELM value (or even a slightly higher value) about 4 
ms after the crash, the $\mathrm{T}_{e}$ gradient accelerates its approach towards the pre-ELM value. When both gradients have approximately reached their final values about $7 \mathrm{~ms}$ after the crash, strong fluctuations of temperature and density gradients around their mean value set in, which may last for several ms. The occurence of the next ELM can vary within this fluctuating phase, which can lead to discharges having two distinct ELM frequencies, with the ELM occuring either right at the gradient recovery or several $\mathrm{ms}$ after the start of the fluctuating phase. The empirical picture to explain this behaviour requires the interplay of different transport channels, the edge ballooning instability alone is not sufficient to explain the observed ELM behaviour. Peeling-ballooning theory suggests the edge current buildup time to be important for the temporal evolution of the ELM cycle, but our experimental findings suggest a more complicated picture. Current diffusion calculations showed that the edge current builds up almost as fast as the gradients, with a delay of less than $1 \mathrm{~ms}$ [7]. This disqualifies a delayed edge current diffusion as a trigger late during the fluctuating phase. The presence of ETG instabilities may explain why the $\mathrm{T}_{e}$ gradient does not increase during the density recovery phase. In the later phase, turbulent instabilities like filaments are supposed to be responsible for the large gradient fluctuations.

\subsection{Interaction of turbulence and geodesic acoustic modes (GAMs) during the L-H transition}

The physics of the L-H transition is still not explained by first principle theory. Turbulence studies by means of Doppler reflectometry recently performed on ASDEX Upgrade [14] shed light on the possible role of geodesic acoustic modes (GAMs) on the formation of the edge transport barrier (ETB). The studies were performed under low density conditions, where the L-H threshold power increases with decreasing density. These conditions were favourable for the measurement of GAMs with Doppler reflectometry. A complicated interplay of GAMs, edge turbulence and mean flow shear could be disentangled. Figure $4 \mathrm{a}$ shows a time trace of the Doppler shift of fluctuations during a discharge phase close to an L-H transition, measured closely inside the separatrix around the position of the $\mathrm{E}_{r}$ minimum. The phases with enhanced Doppler shifts are associated with GAMs, the discharge is in an I-mode phase with confinement characteristics in between L-mode and H-mode. Comparisons of this Iphase with the characteristics of the I-mode phase in Alcator C-Mod [15] are foreseen in the next campaign scheduled from November 2010 till July 2011. The improved confinement in the AUG I-Phase is connected to a negative $\mathrm{E}_{r}$ well that is about a factor of 2 deeper than in the preceding L-mode. Synchronized Doppler spectra for the temporal phases with low and high turbulence levels are shown in figure $4 \mathrm{~b}, \mathrm{c}$ together with pure $\mathrm{L}$ and $\mathrm{H}$-mode cases for comparison in $4 \mathrm{~d}$,e. These figures suggest the following predator-prey picture [16] for the interaction of turbulence, GAMs and equilibrium flows at the L-H transition, which is described in more detail in [14]. Strong turbulence excites GAM oscillations. These oscillations produce a sheared flow, which similar to a pronounced equilibrium flow stabilizes the turbulence and finally the GAM due to the loss of its driver. For these discharge conditions, several phases with low/high turbulence levels alternate. Finally, if the discharges enter into 

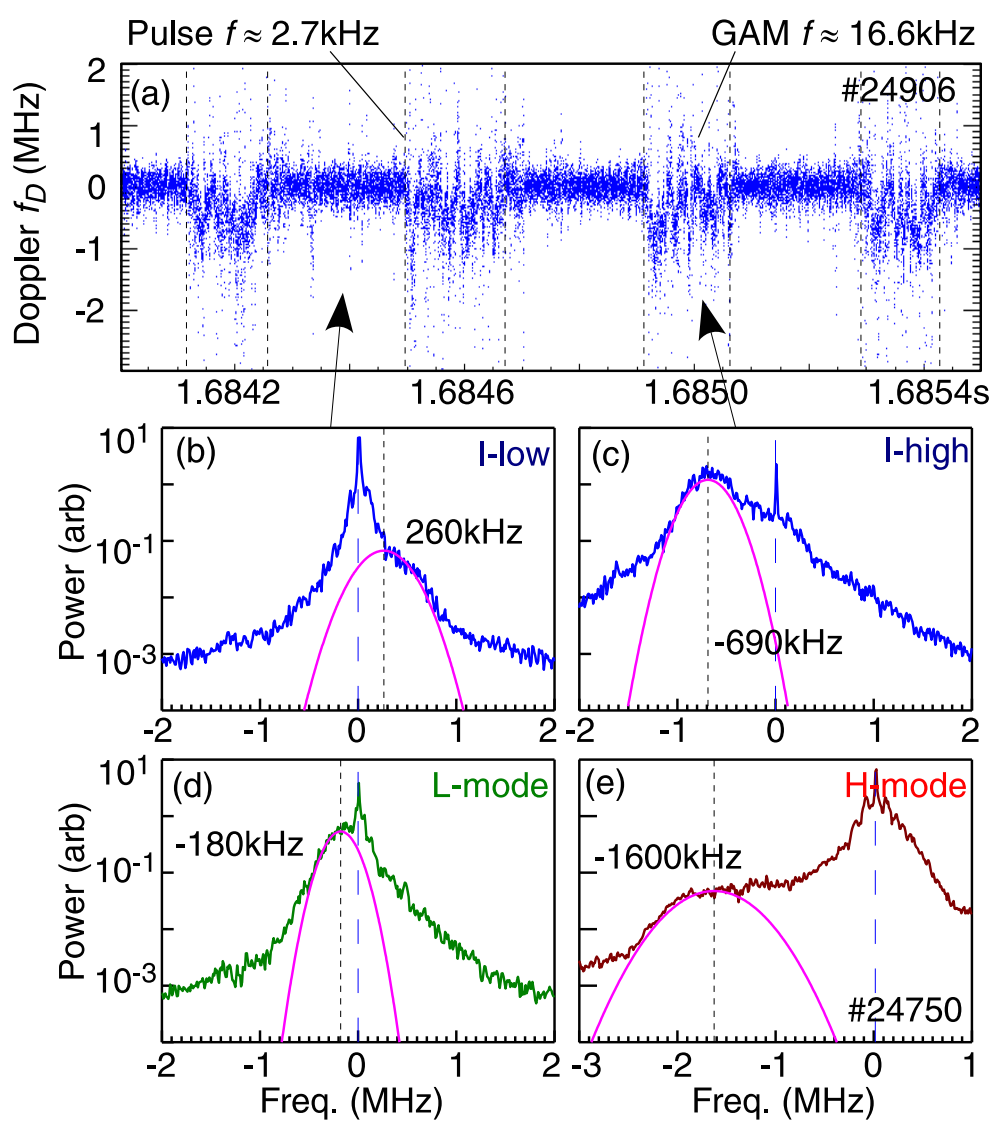

Figure 4. Instantaneous Doppler shift $f_{D}$ time series (a) showing several I-mode pulses with GAM oscillations present, plus synchronized Doppler spectra $S(f)$ from the reduced $(b)$ and enhanced (c) phases. For comparison are also shown an L-mode (d) earlier in the discharge and an H-mode spectrum (e) from similar \# 24570 [14].

H-mode, the sheared equilibrium flow takes over in stabilizing the turbulence. At this point, no GAM is observed any more in the edge region. The intermittent turbulence stabilization may be the crucial ingredient for the transition into the fully developed H-mode. Although these results were obtained for low density conditions, it cannot be excluded that the described mechanism is also valid for any $\mathrm{L}$ to $\mathrm{H}$ transition. On the other hand, the localisation of the measurements in the low density branch of the L-H transition may bring additional physical mechanisms, which are not necessarily present in a higher density regime.

\section{Core transport}

\subsection{Core transport studies with ECRH}

Centrally deposited wave heating is an important tool to avoid accumulation of tungsten ions in the plasma center for standard, high current H-mode discharges in AUG [17]. To improve and to benchmark the theoretical understanding of core transport, dedicated transport studies were performed for different, low plasma current conditions [18] $\left(\mathrm{I}_{p}=0.6 \mathrm{MA}, \mathrm{B}_{t}=-2.5 \mathrm{~T}\right.$, 
$\mathrm{P}_{N B I}=5 \mathrm{MW}, \bar{n}_{e}=5 \times 10^{19} \mathrm{~m}^{-3}$ ). These conditions allowed a strong variation of the $\mathrm{T}_{e} / \mathrm{T}_{i}$ ratio and better experimental parameter characterisation as input for gyrokinetic calculations. While for typical AUG parameters central heating leads to a flattening of the density profiles, a different behaviour can be obtained in lower $\mathrm{I}_{p}$, low density conditions. Figure 5 shows the effect of central ECRH on various plasma profiles under such conditions [18]. Here, even though only $2 \mathrm{MW}$ of ECRH are added to a 5MW NBI heated discharge, a strong rise of the central electron temperature still occurs. Ion temperature and toroidal plasma rotation have been measured with charge exchange recombination spectroscopy (CXRS) on boron ions, which give a lower noise and uncertainty compared to CXRS on carbon for boronized conditions. The change in toroidal rotation caused by the ECRH is remarkable. The central rotation speed is drastically reduced and transport analyses show that diffusion alone can not explain the observed profiles; non-diffusive transport terms must be active. The central ion temperature is also considerably reduced as a consequence of the central electron heating. Similar to the electron density profile the boron density profile peaks moderately with centrally applied ECRH power. Variations of the ECRH deposition radius showed that the pronounced profile changes shown in figure 5 occur only for very central deposition. Theoretical studies [19] in fact explain the contrasting behaviour of different
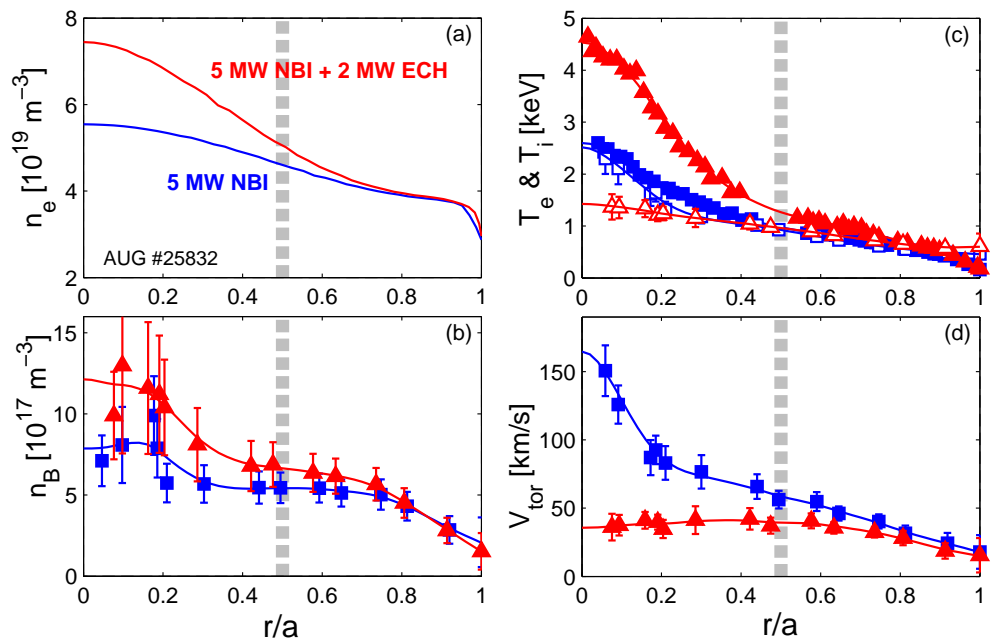

Figure 5. Comparison of low density discharge phases with 5 MW NBI heating with (red) and without (blue) $2 M W$ central ECRH at $I_{p}=0.6 M A$. Shown are radial profiles of a) the electron density, $b$ ) $T_{i}$ (open symbols) and $T_{e}$ (full symbols) from CXRS and ECE diagnostics, $c$ ) the $B^{5+}$ density profile and d) toroidal rotation profile from CXRS. The vertical grey bars indicate the spatial location for the quasi-linear and non-linear transport calculations.

discharge types to central electron heating by the interplay of different control parameters like the $\mathrm{T}_{e} / \mathrm{T}_{i}$ ratio, normalized gradient lengths and collisionality. Numerical calculations reveal that dominant instabilities around mid-radius are found to be ion temperature gradient (ITG) modes during the heating phases without ECRH and with low ECRH power (around 0.6 MW), whereas a transition to dominant trapped electron modes (TEM) occurs in the phases with high ECRH power (around $2 \mathrm{MW}$ ). Figure 6 compares measured normalized 
electron density inverse gradient lengths with GS2 [20] quasi-linear and GYRO [21] nonlinear calculations for conditions corresponding to profiles shown in figure 5. Good agreement is found for the dependence on the $\mathrm{T}_{e} / \mathrm{T}_{i}$ temperature ratio. In fact, the dependence of $\mathrm{R} / \mathrm{L}_{n e}$ on all relevant experimental parameters $\left(\mathrm{R} / \mathrm{L}_{T e}, \mathrm{R} / \mathrm{L}_{T i}\right.$, collisionality) appears to be well ordered by the real frequency of the most unstable mode [22], see figure 6b. As shown in figure
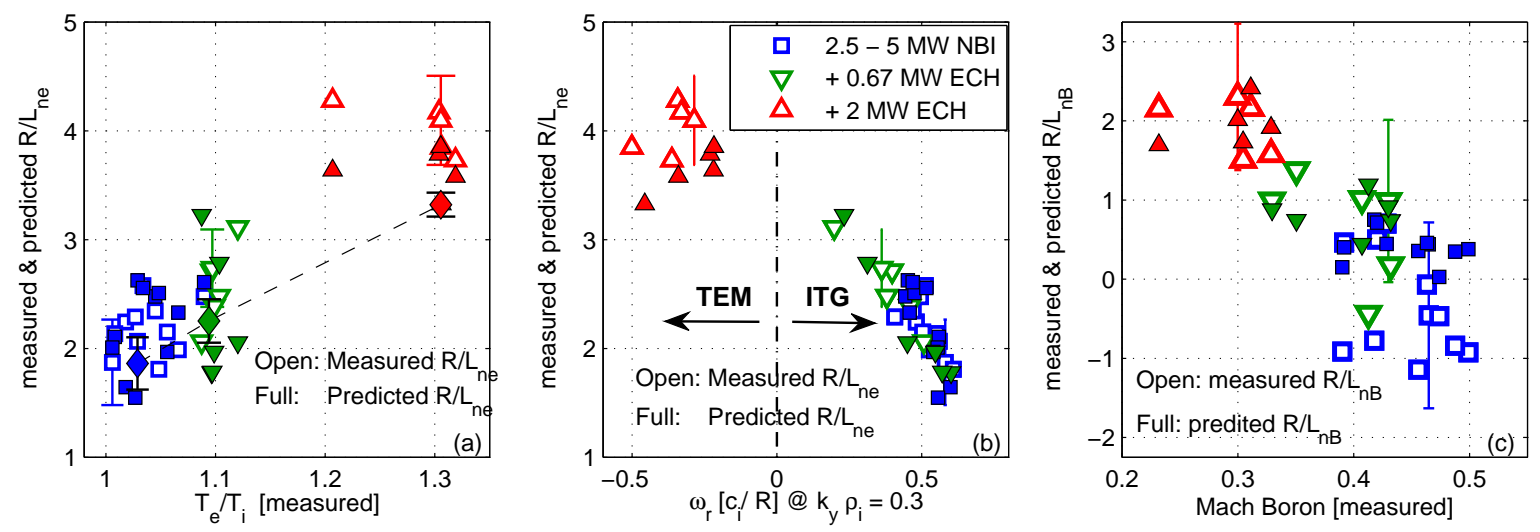

Figure 6. Analysis of representative cases of the three heating phases corresponding to figure 5 at half radius. a) Comparison of predicted quasi-linear GS2 (full symbols with squares, triangles pointing down and up), nonlinear GYRO (diamonds connected by a dashed line) and measured (open symbols) values of $R / L_{n e}$ as a function of the temperature ratio $T_{e} / T_{i} . b$ ) Predicted and measured $R / L_{n e}$ versus the real frequency of the most unstable mode at $k_{y} \rho_{i}=$ 0.3. c) Normalized inverse gradient length $R / L_{n B}$ of $B^{5+}$ ions versus the toroidal Mach number [19]. This figure has corrected an error in the theoretical calculations shown in corresponding IAEA viewgraph Fig. 6 in [23]. The correct mode rotation in the high ECRH power phase is negative, indicating dominant TEM instabilities.

6c, the change of the normalized logarithmic gradients $R / L_{n B}$ of the boron densities is also satisfactorily predicted. The boron profiles are much less peaked compared to the electron density at half radius, or even slightly locally hollow. The numerical predictions reproduce the experimentally observed dependence, with lower values of $R / L_{n B}$ in the NBI only heated phases, and with larger values of $R / L_{n B}$ with increasing ECH power, although locally hollow profiles, that is negative values of $R / L_{n B}$, are not predicted by the simulations. In order to reproduce the experimentally observed behaviour of the boron density profiles, the toroidal rotation and its radial gradient [24] turned out to be an important ingredient in the modelling of the turbulent boron ion transport. In the event that this contribution is neglected, the values of $R / L_{n B}$ are predicted not to vary significantly in the different heating phases, in disagreement with the experimental observations [19].

\subsection{Effect of nitrogen seeding on plasma performance}

Feedback-controlled nitrogen seeding is used routinely in high power discharges to protect the divertor tungsten coatings from thermal overload [2]. As a positive side effect, improved energy confinement and an increased ELM frequency at shorter ELM duration have been 

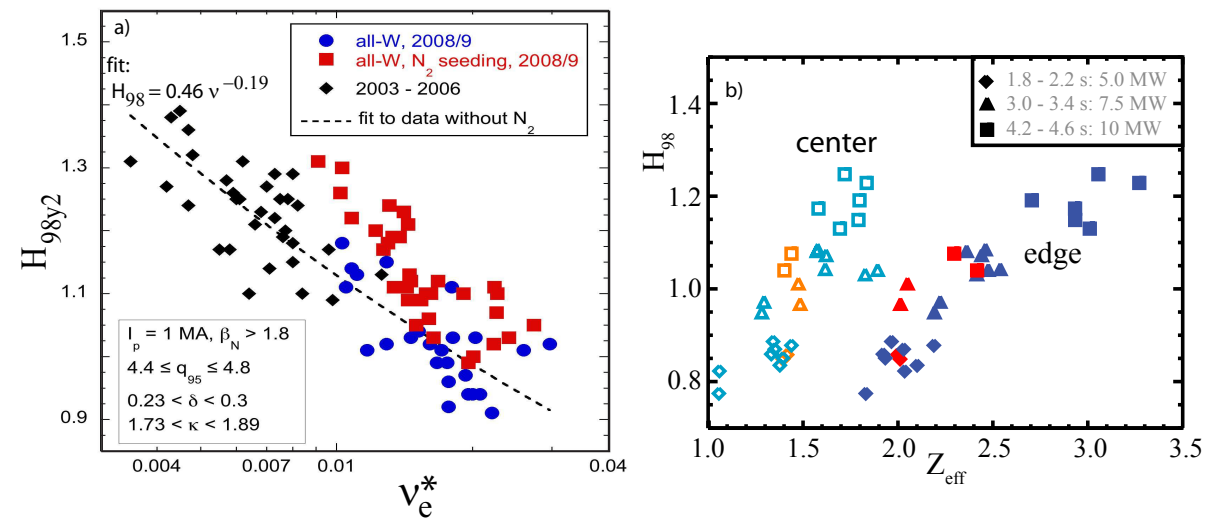

Figure 7. a) $H_{98(y, 2)}$ confinement factor vs. collisionality for different H-mode discharges with and without nitrogen seeding. $b) \mathrm{H}$-factor $H_{98(y, 2)}$ for discharges with /without (blue/red) nitrogen seeding versus $Z_{\text {eff }}$ at $\rho_{\text {pol }}=0.2 / 0.9$ (open/full symbols) [25].

observed [17]. The nitrogen puff leads mainly to increased divertor radiation, while the total radiation inside the separatrix stays approximately constant. The confinement improvement, as shown in figure $7 \mathrm{a}$, is related to increased temperatures at the pedestal top and in the plasma core, while the electron density profile is hardly effected by the presence of nitrogen. Analysis of $\mathrm{Z}_{\text {eff }}$ profiles derived from bremsstrahlung revealed a close correlation of the $\mathrm{H}_{98}$ confinement qualifier with $\mathrm{Z}_{\text {eff }}$ [25], as shown in figure $7 \mathrm{~b}$. These results suggest a simple, empirical picture for the confinement behaviour, based on the observation that impurity concentration profiles are hollow: Assuming that the total edge pressure is limited by an instability, the ion dilution by the presence of impurities results in a higher pedestal temperature. If the core temperature profile is stiff, which is a good approximation for AUG conditions, the increased pedestal temperature propagates to the plasma center. Hollow impurity profiles lead to a decrease of dilution towards the center and hence result in an increase of the total pressure and $\mathrm{H}_{98}$ caused by the higher ion density. In addition to the pedestal dilution effect, changes in core transport are expected. Gyrokinetic calculations suggest changes of the critical gradient as well as in the degree of temperature profile stiffness, both effects tend to cancel in their overall effect on confinement [26]. Clearly, further theorybased calculations are required for a quantitative understanding of the pedestal response to the nitrogen seeding.

\subsection{Fast particle losses}

Fast particle transport through the plasma and their losses towards plasma facing components may affect the fusion rate, the current drive location and efficiency and the integrity of vessel structures in a fusion reactor. Various MHD modes may contribute to fast particle transport, like Alfvén cascades (ACs) and toroidal Alfvén eigenmodes (TAEs). Location and structure of the modes are determined by various diagnostics in ASDEX Upgrade, like soft X-ray diodes [28], reflectometry [29], collective Thomson scattering [30] and fast ECE imaging 

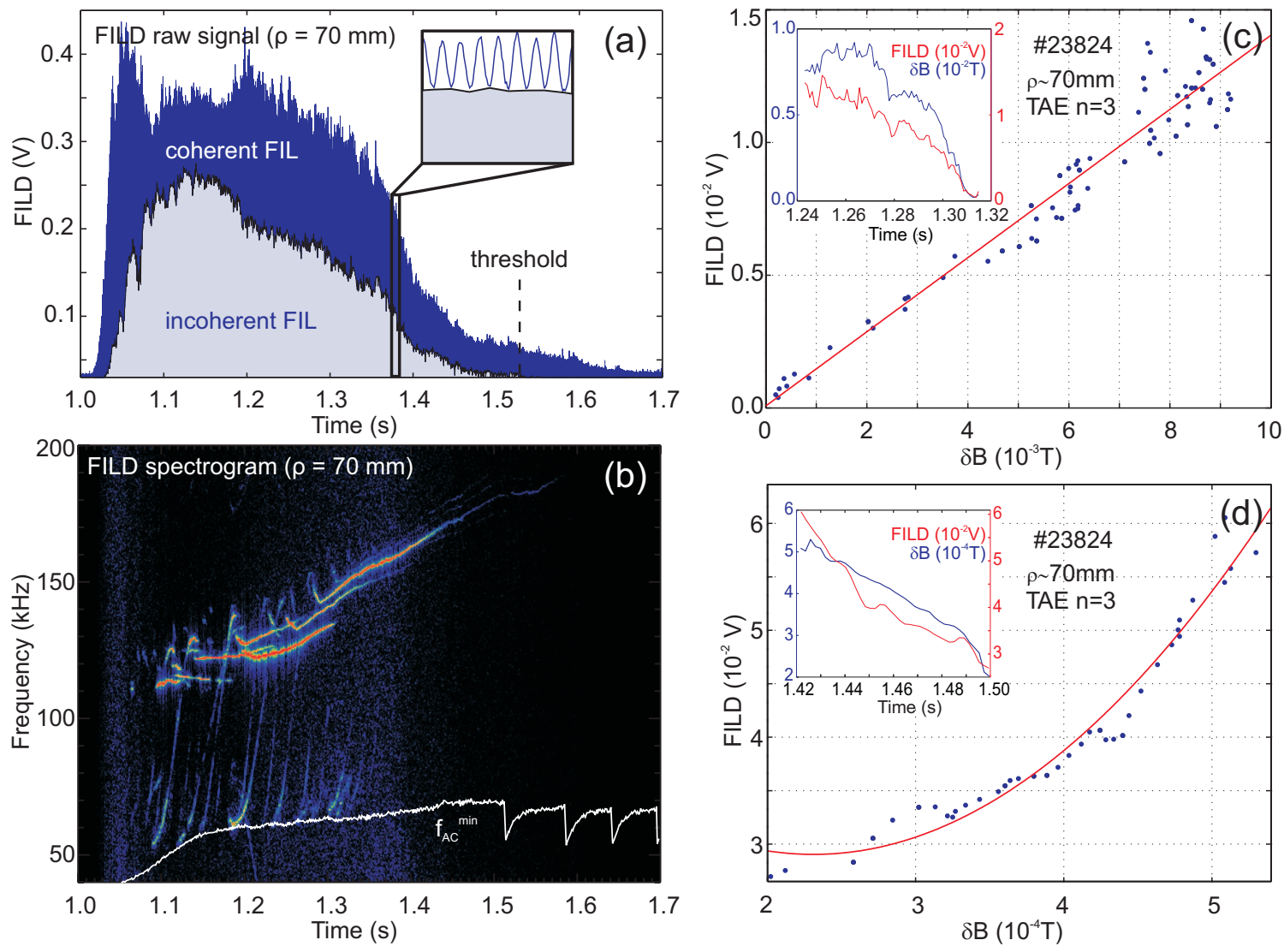

Figure 8. a) Raw signal of the fast ion loss detector during a pulse with strong MHD activity. b) Spectrogram showing AC and TAE activity. The geodesic frequency $f_{A C}^{\min }[27]$ is indicated in white for reference. c) Linear dependence of coherent FIL signal on magnetic fluctuating amplitude $\delta B$ interpreted as convective losses. $d$ ) Nonlinear (offset-quadratic) dependence of incoherent FIL signal on $\delta B$ interpreted as conductive losses.

[31] measurements. Particle losses are detected by a fast ion loss detector (FILD) [32]. Figure 8 shows the raw signal and spectrograms obtained by the FILD detector during a discharge with AC and TAE activity [33]. The raw signal exhibits a fluctuating component, which is closely related to the frequency signatures of the MHD activities as observed by soft X-ray and Mirnov coils. This component of the fast ion losses is related to a convective loss mechanism, where the perturbing field amplitude leads to the direct loss of particles. Analysis with the HAGIS code revealed that the lost particles are not those which dominate the drive [34]. The FILD raw signal also exhibits a component which is time-independent on the MHD mode time scales (lower envelope in figure 8a). This component is attributed to a diffusive loss term, invoking more than one perturbation. The different behaviour of the convective and diffusive losses is shown in figure 8c,d, where a linear and a quadratic dependence on the perturbed field amplitude is found. Investigations of turbulent fast ion transport by means of fast ion $\mathrm{D}_{\alpha}$ (FIDA) and collective Thomson scattering (CTS) diagnostics have started [33]. 


\section{Power exhaust and plasma-wall interaction}

\subsection{High power exhaust experiments}

The ratio of separatrix power flux and major radius, $\mathrm{P} / \mathrm{R}$, is a performance parameter for divertor power exhaust [35] and a measure for the effort required to keep the divertor peak power load below a certain critical level. High power experiments were conducted with feedback-controlled nitrogen seeding to keep the target peak heat flux at a moderate level. Nitrogen is puffed at several toroidal locations in the X-point region, using proportionalintegral (PI) feedback on the outer divertor temperature derived from shunt measurements of the inter-ELM thermoelectric divertor current [36]. Figure 9 shows time traces of a discharge with $20 \mathrm{MW}$ heating power, including about $1 \mathrm{MW}$ central ECRH in $\mathrm{O} 2$ mode (central electron density is above X2 cut-off density). Good energy confinement $\left(\mathrm{H}_{98(y, 2)}=1\right)$ could be obtained simultaneously with a low impurity content of the core plasma $\left(\bar{Z}_{\text {eff }}<1.8\right)$. Although the discharge used also strong deuterium puffing to enhance SOL/divertor radiation, the corresponding confinement degradation is compensated by the improvement caused by nitrogen. The radiation distribution from bolometer tomography is shown in the middle of figure 9, about half of the radiated power is emitted in the SOL and divertor. It is worthwhile to compare the measured divertor and SOL radiation of $\approx 6 \mathrm{MW}$ with a simple analytical estimate based on a radiative loss model coupled to classical parallel electron heat conduction [37]. SOL and divertor are approximated as a single flux tube with the radial width equal to the power decay length $\lambda_{p}$ in the outer midplane. Electron pressure conservation is assumed along the flux tube.

The radiated heat flux is calculated as the integral of the weighted radiative loss function, $\mathrm{L}_{z}$, where non-Coronal effects are introduced via the parameter $n_{e, d i v} \tau_{N}$, where $n_{e, d i v}$ is the characteristic divertor electron density and $\tau_{N}$ the residence time of nitrogen in the divertor plasma [38]. With $\lambda_{p}=510^{-3} \mathrm{~m}$ about $5 \mathrm{MW}$ radiated power is predicted for the conditions of the discharge shown in figure 9, well in line with the experiment. A factor of 4 enhancement above the Coronal radiation level is obtained for these conditions, a lower level of enhancement has to be assumed for reactor conditions with higher divertor density and longer residence time $\tau_{N}$. Nitrogen seeding does not significantly enhance the radiation inside the pedestal. To control the power flux through the pedestal independently from the divertor heat flux, multi-species impurity seeding has to be applied. Initial experiments have been performed with combined $\mathrm{N}$ and Ar injection [39].

\subsection{Tungsten melt experiment}

So far, tungsten erosion and transport studies concentrated on normal H-mode operation. For these conditions, the outer limiters have been identified as the tungsten source which is most important for the observed core tungsten concentration [12]. Despite the higher absolute W source strength in the divertor, its good W retention capability leads to a relatively small core penetration. For preparation of future high power devices with a tungsten divertor, 


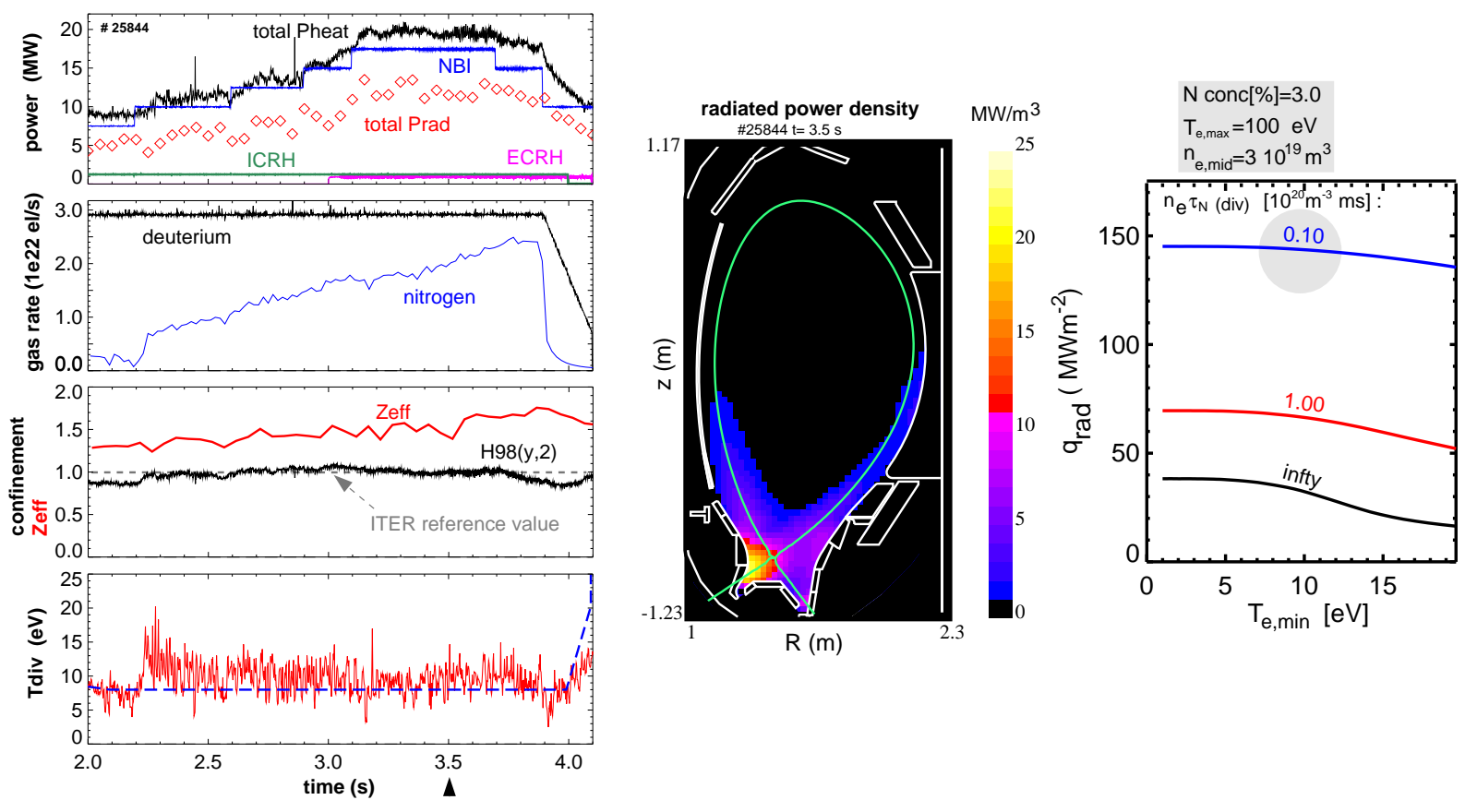

Figure 9. High $P / R$ discharge with divertor power load control by nitrogen seeding in ASDEX Upgrade. left: Time traces of various plasma parameters. The strong deuterium puff allows for low $Z_{\text {eff }}$ in the core plasma, while the confinement improvement due to nitrogen maintains $H$-mode confinement with $H_{98, y 2}=1$. Tdiv is derived from a shunt measurement of the inter-ELM thermoelectric divertor current and is a proxy for the outer divertor electron temperature. Middle: radiation distribution from tomographic bolometer reconstruction at $t=3.5 \mathrm{~s}$. right: estimated maximum radiated parallel power flux density from simple analytical model and ADAS data. 'infty' refers to Coronal conditions, non-Coronal enhancement is expected for the present conditions (grey area) corresponding to $n_{e} \tau_{N}=10^{16} \mathrm{~m}^{-3} \mathrm{~s}$.

melting events have to be studied, which can occur either during abnormal events or due to imperfections of tile alignments. To simulate an abnormal tungsten melting in the divertor, small $\mathrm{W}$ pin probes were inserted into the divertor plasma by means of a manipulator for attached L-mode and detached H-mode conditions [41]. The experiments were performed under well diagnosed and stable plasma conditions, results are shown in figure 10. Main chamber injection of tungsten was done by laser blow-off (LBO) later in the discharge for the same plasma conditions, allowing the comparison of the rise of the core tungsten concentration for the different release locations. Thus, the divertor screening for molten $\mathrm{W}$ injection was measured. For the $\mathrm{H}$-mode case, tungsten core penetration probabilities (probability for a released atom to reach the core plasma) of 1/260 and 1/11 were derived for the divertor and the main chamber, respectively [40]. The experiments were accompanied by numerical simulations with the 3D edge code EMC3-Eirene [42]. A high divertor screening could be reproduced, albeit a strong dependence of the penetration probability from the distance of the injection position to the strike point was found in the modelling. 

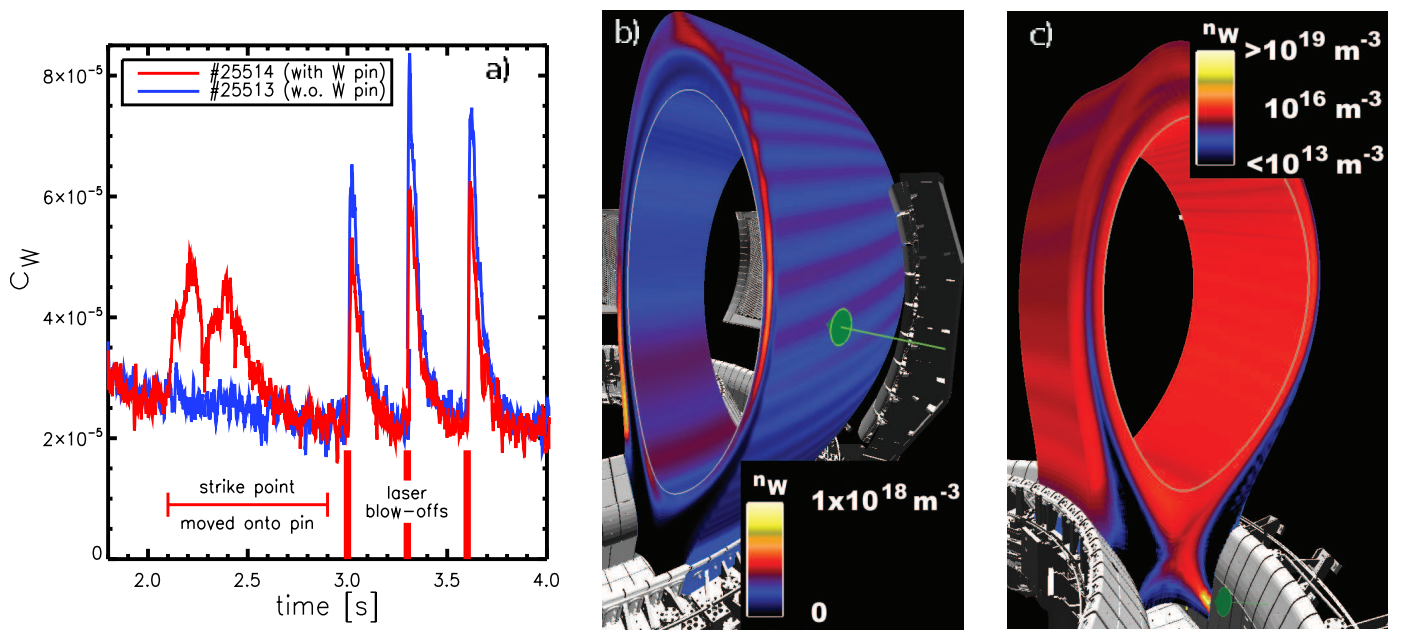

Figure 10. W concentration measurements and EMC3-Eirene modelling of a $W$ melt experiment. a) measured $W$ concentration for $W$ melting and W-LBO. b) EMC3-Eirene calculation of $W$ injection in the midplane by laser blow-off. c) EMC3-Eirene calculation for ablation in the divertor at the strike point position (log scale) [40].

\subsection{Disruption mitigation}

Disruptions have been successfully mitigated with massive gas injection (MGI) [43] and disruption avoidance or delay by off-axis ECRH has been demonstrated for high $\beta_{N}$ discharges [44] [45]. During MGI mitigated discharges, newly installed AXUV (Absolute X-ray and UV diode detectors) bolometer arrays allowed the study of fast radiation events including the VUV radiation, which is blocked by the filters of the soft X-ray diagnostic. The temporal and spatial evolution of the radiating zone is particularly important, since large asymmetries may lead to excessive wall loads in ITER. Figure 11 shows the propagation of the radiating zone during a fast plasma shutdown by massive gas injection, measured by poloidal fans of AXUV diodes in the sector of the gas valve and on the opposite side of the torus. A poloidal propagation of filamentary radiating layers is clearly observed. On the opposite side of the torus, the radiation rise is substantially delayed. Impurities are supposed to reach the high field side predominantly by streaming parallel to the field lines, plus some additional drift effect. The emissivity front moves towards the plasma center, does not penetrate that far, but reaches the surface with $\mathrm{q} \approx 2$ within $2 \mathrm{~ms}$; afterwards the thermal quench sets in. The experiments with one MGI valve generally show a highly anisotropic radiating layer. To reduce this localization, the simultaneous use of several MGI valves may be required.

Disruption avoidance or at least substantial delay has been achieved by localized injection of 1.5 MW ECRH onto the $\mathrm{q}=2$ resonant surface in a high $\beta_{N}$ scenario [45]. Like the MGI, in standard $\mathrm{I}_{p} \geq 1 \mathrm{MA}$ operation the ECRH is triggered by the locked mode monitor precursor signal of the 2/1 mode. For suitable scenarios, ECRH injection can be considered as a complementary technique to MGI for disruption control. 

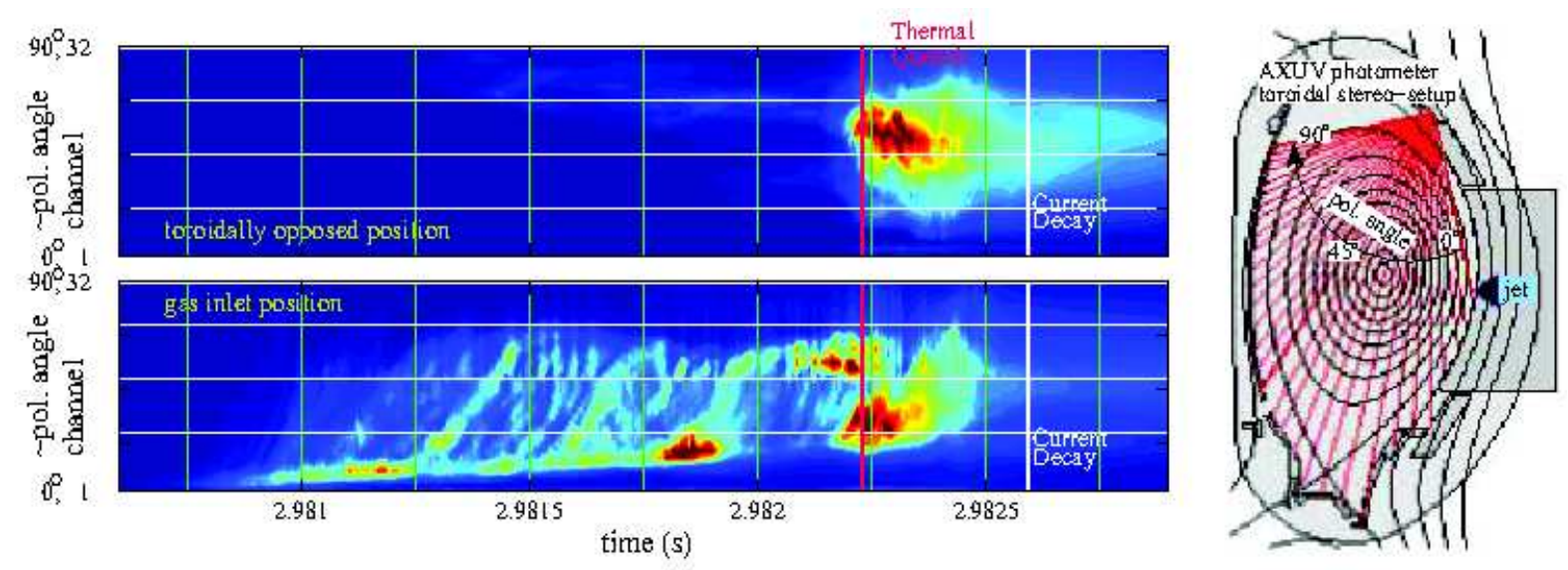

Figure 11. Temporal and spatial development during a neon-MGI mitigated disruption in a toroidal position opposite to the MGI valve and in sector of the MGI valve for \# 24442 [43]. The right figure shows the geometry of the viewing lines, from this, a poloidal motion of filaments over the plasma top towards the high field side can be inferred in the gas valve sector.

\subsection{Plasma-wall interaction with nitrogen seeding}

Fuel retention and heat removal capabilities of tungsten depend strongly on its microscopic characteristics in the near-surface layers. A modification of the material properties is expected due to the storage of nitrogen, which is now routinely used in high power discharges. The formation of a tungsten nitride layer with a 1:1 atomic ratio in the first few $\mathrm{nm}$ below the surface had been observed in laboratory experiments [46]. Nitrogen particle balance in AUG seeded discharges revealed even an order of magnitude higher nitrogen storage of about $10^{21} \mathrm{~N}$ atoms $/ \mathrm{m}^{2}$. The higher storage is expected to be caused by surface roughness and co-deposition with boron and carbon in local recessions in the tungsten surface [38]. Laboratory studies showed a reduction of the tungsten sputtering yield by nitrogen compared to a pure tungsten surface. Collisions of impacting ions with embedded nitrogen atoms are supposed to reduce the sputtering yield for tungsten. Figure 12 shows tungsten sputtering yields measured in AUG for conditions of dominant sputtering by nitrogen. Within the considerable uncertainties, the measured yields agree with the TRIDYN code [47] predictions without a modification of the yields due to nitrogen storage. Surface analysis and ion beam experiments with tokamak samples will be required to resolve the differences between the 'ideal' laboratory and tokamak surface conditions.

\section{Conclusions and outlook}

Re-establishment of its full flywheel-generator capabilities allowed us to operate the fulltungsten ASDEX Upgrade tokamak with up to $20 \mathrm{MW}$ heating power. New results have been obtained in particular on the pedestal structure and transport, the $\mathrm{L}-\mathrm{H}$ transition, core transport, fast ion losses, high power operation with radiative cooling and plasma-wall 


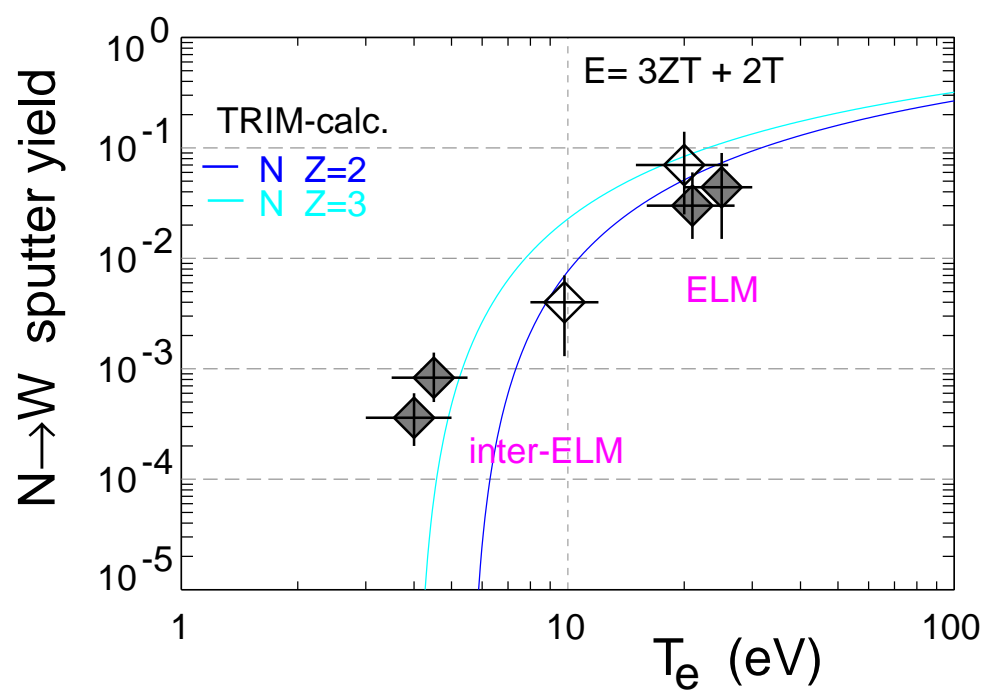

Figure 12. Measured tungsten sputtering yields for nitrogen seeded discharges versus an analytic fit of TRIDYN calculations for $\mathrm{N}^{2+}$ and $\mathrm{N}^{3+}$ ions. Solid symbols represent conditions close to nitrogen-saturated tungsten divertor surface. The open symbols are not nitrogen-saturated [38].

interaction. After installation of the first set of 8 resonant magnetic perturbation (RMP) coils, AUG resumed plasma operation in December 2010. Two new gyrotrons will increase the ECRH capability up to $5 \mathrm{MW}$. Characterisation of a modified ICRF antenna will be used to validate antenna models developed with HFSS and TOPICA codes, aiming towards an antenna design leading to reduced tungsten erosion. Power exhaust studies will aim at the integration of impurity seeding and RMP controlled ELMs in the tungsten PFC environment.

References presented below as papers at this conference are available online at http://wwwnaweb.iaea.org/napc/physics/FEC/FEC2010/html/index.htm

[1] NEU, R. et al., Plasma Physics and Controlled Fusion 49 (2007) B59.

[2] KALLENBACH, A. et al., Nuclear Fusion 49 (2009) 045007.

[3] KALlENBACH, A. et al., Plasma Physics and Controlled Fusion 47 (2005) B207.

[4] BOBKOV, V. V. et al., Journal of Nuclear Materials 363-365 (2007) 122.

[5] NOTERDAEME, J.-M. et al., Advances in ICRF physics and technology on ASDEX Upgrade, IAEA-CN180/EXM/P7-21 .

[6] HÖHNLE, H. et al., Extension of the ECRH operational space with O2 and X3 heating schemes to control W accumulation in ASDEX Upgrade, IAEA-CN-180/P7-25 .

[7] BURCKHART, A. et al., Plasma Phys. Controlled Fusion (2010) 105010.

[8] KURZAN, B. et al., Transport in-between edge localized modes in the pedestal of ASDEX Upgrade, IAEA-CN-180/EXC/P3-03 .

[9] WOLFRUM, E. et al., Plasma Physics and Controlled Fusion 51 (2009) 124057 (9pp).

[10] CONWAY, G. D. et al., Plasma Physics and Controlled Fusion 46 (2004) 951.

[11] PÜTTERICH, T. et al., ELM flushing and impurity transport in the H-mode edge barrier in ASDEX Upgrade, J. Nucl. Mat. in press (2011), doi:10.1016/j.jnucmat.2010.09.052 .

[12] DUX, R. et al., Nucl. Fusion 51 (2011) 053002.

[13] BURRELL, K. H. et al., Plasma Phys. Controlled Fusion 44 (2002) A253.

[14] CONWAY, G. et al., Phys. Rev. Lett. 106 (2011) 065001. 
[15] WHYTE, D. et al., Nucl. Fusion 50 (2010) 105005.

[16] KIM, E. and DIAMOND, P., Phys. Rev. Lett. 90 (2003) 185006.

[17] GRUBER, O. et al., Nuclear Fusion 49 (2009) 115014.

[18] MCDERMOTT, R. et al., Plasma Phys. Controlled Fusion 53 (2011) 035007.

[19] ANGIONI, C. et al., Nucl. Fusion 51 (2011) 023006.

[20] KOTSCHENREUTHER, M. et al., Comput. Phys. Commun. 88 (1995) 128.

[21] CANDY, J. and WALTZ, R., J. Comput. Phys. 186 (2003) 545.

[22] FABLE, E. et al., Plasma Phys. Controlled Fusion 52 (2010) 015007.

[23] KALLENBACH, A. et al., Overview of ASDEX Upgrade results, IAEA-CN-180/OV/3-1 .

[24] CAMENEN, Y. et al., Physics of Plasmas 16 (2009) 062501.

[25] RATHGEBER, S. K. et al., Plasma Physics and Controlled Fusion 52 (2010) 095008.

[26] SCHWEINZER, J. et al., Confinement of Improved H-modes in the all-tungsten ASDEX Upgrade, IAEACN-180/EXC/P2-07.

[27] BERK, H. et al., Physical Review Letters 87 (2001) 185002.

[28] PIOVESAN, P. et al., Nuclear Fusion 48 (2008) 065001.

[29] da Graca, S. et al., Plasma Physics and Controlled Fusion 49 (2007) 1849.

[30] MEO, F. et al., Review of Scientific Instruments 79 (2008) 10 E501.

[31] CLASSEN, I. G. J. et al., Review of Scientific Instruments 81 (2010) 10 D929.

[32] GARCÍA-MUñOZ, M. et al., Review of Scientific Instruments 80 (2009) 053503.

[33] GARCÍA-MUñOZ, M. et al., Fast-ion transport induced by Alfven eigenmodes in ASDEX Upgrade, submitted to Nucl. Fusion (February 2011) .

[34] LAUBER, P. et al., Damping, drive and non-linear effects of kinetic low-frequency modes in tokamaks, IAEA-CN-180/THW/2-2Ra .

[35] LACKNER, K., Comments Plasma Phys. Controlled Fusion 15 (1994) 359.

[36] KALlENBACH, A. et al., Plasma Physics and Controlled Fusion 52 (2010) 055002.

[37] LACKNER, K. and SCHNEIDER, R., Fusion Eng. and Design 22 (1993) 107.

[38] KALLENBACH, A. et al., Plasma surface interactions in impurity seeded plasma, J. Nucl. Mat. in press (2011), doi:10.1016/j.jnucmat.2010.11.105 .

[39] NEU, R. et al., Power and particle exhaust control in all-W ASDEX Upgrade, IAEA-CN-180/EXD/P3-24

[40] LUNT, T. et al., 3D modeling of the ASDEX Upgrade edge plasma exposed to a localized tungsten source by means of EMC3-Eirene, J. Nucl. Mat. in press (2011), doi:10.1016/j.jnucmat.2010.11.009.

[41] KRIEGER, K. et al., J. Nucl. Mat. in press (2011), doi:10.1016/j.jnucmat.2010.12.229.

[42] FENG, Y. et al., Contrib. Plasma Phys. 44 (2004) 57.

[43] PAUTASSO, G. et al., Contribution of ASDEX Upgrade to disruption studies for ITER, submitted to Nucl. Fusion (December 2010) .

[44] ESPOSITO, B. et al., Nuclear Fusion 49 (2009) 065014.

[45] ESPOSITO, B. et al., Avoidance of disruptions at high $\beta_{N}$ in ASDEX Upgrade with off-axis ECRH, submitted to Nucl. Fusion (January 2011) .

[46] SCHMID, K. et al., Nucl. Fusion 50 (2010) 025006.

[47] MÖLLER, W. et al., Comput. Phys. Commun. 51 (1988) 355. 